\title{
Vector solitons with an embedded domain wall
}

\author{
P. G. Kevrekidis, ${ }^{1, *}$ H. Susanto, ${ }^{2, \dagger}$ R. Carretero-González, ${ }^{3, \ddagger}$ B. A. Malomed, ${ }^{4, \S}$ and D. J. Frantzeskakis ${ }^{5, \|}$ \\ ${ }^{1}$ Department of Mathematics and Statistics, University of Massachusetts, Amherst, Massachusetts 01003-4515, USA \\ ${ }^{2}$ Department of Applied Mathematics, University of Twente, P.O. Box 217, 7500 AE Enschede, The Netherlands \\ ${ }^{3}$ Nonlinear Dynamical Systems Group, Department of Mathematics and Statistics, and Computational Science Research Center, \\ San Diego State University, San Diego, California 92182-7720, USA \\ ${ }^{4}$ Department of Interdisciplinary Studies, Faculty of Engineering, Tel Aviv University, Tel Aviv 69978, Israel \\ ${ }^{5}$ Department of Physics, University of Athens, Panepistimiopolis, Zografos, Athens 15784, Greece
}

(Received 24 July 2005; published 9 December 2005)

\begin{abstract}
We present a class of soliton solutions to a system of two coupled nonlinear Schrödinger equations, with an intrinsic domain wall (DW) which separates regions occupied by two different fields. The model describes a binary mixture of two Bose-Einstein condensates (BECs) with interspecies repulsion. For the attractive or repulsive interactions inside each species, we find solutions which are bright or dark solitons in each component, while for the opposite signs of the intraspecies interaction, a bright-dark soliton pair is found (each time, with the intrinsic DW). These solutions can arise in the context of discrete lattices, and most of them can be supported in continuum settings by an external parabolic trap. The stability of the solitons with intrinsic DWs is examined, and the evolution of unstable ones is analyzed. We also briefly discuss the possibility of generating such families of solutions in the presence of linear coupling between the components, and an application of the model to bimodal light propagation in nonlinear optics.
\end{abstract}

DOI: 10.1103/PhysRevE.72.066604

PACS number(s): 05.45.Yv, 03.75.Lm

\section{INTRODUCTION}

In the past few years, the experimental and theoretical studies of Bose-Einstein condensates (BECs) have drawn a great deal of interest $[1,2]$. In particular, the dynamics of multicomponent BECs was studied in detail. Such multispecies condensates have arisen as mixtures of different spin states in ${ }^{87} \mathrm{Rb}[3,4]$ and ${ }^{23} \mathrm{Na}[5]$ condensates. In addition, many theoretical studies were devoted to mixtures of different bosonic atomic species, such as $\mathrm{Na}-\mathrm{Rb}[6,7], \mathrm{K}-\mathrm{Rb}$ [8,9], $\mathrm{Cs}-\mathrm{Rb}$ [10], and $\mathrm{Li}-\mathrm{Rb}$ [11]. Experimental realization of a two-species BEC, namely the ${ }^{41} \mathrm{~K}-{ }^{87} \mathrm{Rb}$ mixture, has been reported [12]; a mixture of ${ }^{7} \mathrm{Li}$ and ${ }^{133} \mathrm{Cs}$ was also investigated in an experiment [13], but without driving it into the BEC state.

A model providing a very accurate description of the BEC-mixture dynamics is based on a system of coupled Gross-Pitaevskii equations (GPEs) for the mean-field singleatom wave function of each component, which takes into account the self- and cross-interactions between the species. Numerous issues have been considered in this framework. Among them are ground-state solutions [6,14,15], smallamplitude excitations [7,16], formation of domain walls (DWs) between immiscible species [17-19], bound states of dark-bright [20] and dark-dark [21], dark-gray, bright-gray, bright-antidark and dark-antidark [22] complexes of solitary waves, spatially periodic states [23], modulated amplitude

\footnotetext{
*Electronic address: kevrekid@math.umass.edu

†Electronic address: h.susanto@math.utwente.nl

†URL: http://nlds.sdsu.edu/

${ }^{\S}$ Electronic address: malomed@eng.tau.ac.il

"Electronic address: dfrantz@cc.uoa.gr
}

waves [24], and various effects produced by a linear interspecies coupling between the components [23,25] (which is possible in the case of two different spin states of the same atomic species, with the linear coupling induced by a spinflipping electromagnetic wave), including an effect on DWs in the case of immiscibility [26].

In the present work, we suggest another type of twocomponent soliton structures, which exists only when the interspecies interaction is repulsive, while the intraspecies interactions may be both attractive, or both repulsive, or even mixed. The repulsion between the species allows us to construct DW configurations between the species. In the case of attractive self-interactions in each component, the DW will be between two bright solitons, one in each species. With repulsive self-interactions, the DW will separate dark solitons (rather than ground states, as in Ref. [17,18]). Finally, for the mixed interactions, on two sides of the DW we will find a bright soliton in one component and a dark soliton in the other. For such configurations to be stationary, the repulsion between the species needs to be balanced by a confining force. One possibility for that, which we examine in this work, is provided by a deep optical lattice (OL), which, asymptotically, gives rise to a system of coupled discrete GPEs [27-29]. Another possibility is provided by the customary magnetic trapping (i.e., an external parabolic potential), which is a (typically) necessary ingredient of experiments with BECs $[1,2]$. Finally, we will also briefly consider the case without the external trap, but with linear coupling between the components.

A realization of the system in terms of nonlinear optics is quite possible too. In that case, the two species represent either two orthogonal polarizations of light, or two signals with different carrier wavelengths [30]. In the former case, the linear coupling can also be implemented, by twisting the 
waveguides or elliptically deforming them, in the case of linear or circular polarizations, respectively.

The presentation is structured as follows. In Sec. II, we describe the setup and methods to be used for the numerical analysis of relevant solutions. In Sec. III, we study solutions in the discrete coupled-GPE model, and in Sec. IV we consider its continuum analog in the presence of the magnetic trapping. In Sec. V, we deal with the case of the linear coupling between the components. The results of the work are summarized in Sec. VI. In two Appendixes, we present analytical calculations for properties of the solutions as functions of the system's parameters, based on the variational approximation (VA); these predictions are directly compared with numerical results.

\section{THE MODELS AND SETUP}

The present work is based on two basic models, viz., a discrete one and its continuous counterpart. The former model is based on two coupled discrete nonlinear Schrödinger (NLS) equations,

$$
\begin{aligned}
& i \partial_{t} \psi_{1, n}=\left(-C \Delta_{2}+g_{11}\left|\psi_{1, n}\right|^{2}+g_{12}\left|\psi_{2, n}\right|^{2}\right) \psi_{1, n}, \\
& i \partial_{t} \psi_{2, n}=\left(-C \Delta_{2}+g_{12}\left|\psi_{1, n}\right|^{2}+g_{22}\left|\psi_{2, n}\right|^{2}\right) \psi_{2, n},
\end{aligned}
$$

which describe a binary mixture of BECs trapped in a deep OL [2], as well as beam dynamics in optical waveguide arrays [30]. The real constant $C$ accounts for the tunneling coefficient between adjacent troughs in the OL potential, or between adjacent waveguides in the array, and $\Delta_{2}$ is the discrete Laplacian (i.e., the second difference) $\Delta_{2} \psi_{i, n}=\left(\psi_{i, n+1}\right.$ $\left.+\psi_{i, n-1}-2 \psi_{i, n}\right)$. Equation (1) conserves the Hamiltonian and the norm,

$$
P \equiv \frac{1}{2} \sum_{n}\left(\left|\psi_{1, n}\right|^{2}+\left|\psi_{2, n}\right|^{2}\right) .
$$

The second model is the continuous counterpart of the system (1), which also incorporates an external trapping potential $V(x)[1]$ :

$$
\begin{aligned}
& i \partial_{t} \psi_{1}=\left[-\partial_{x}^{2}+g_{11}\left|\psi_{1}\right|^{2}+g_{12}\left|\psi_{2}\right|^{2}+V(x)\right] \psi_{1}, \\
& i \partial_{t} \psi_{2}=\left[-\partial_{x}^{2}+g_{12}\left|\psi_{1}\right|^{2}+g_{22}\left|\psi_{2}\right|^{2}+V(x)\right] \psi_{2} .
\end{aligned}
$$

In these equations, $\psi_{1}$ and $\psi_{2}$ describe the mean-field wave functions of the two-component BEC, or, in the context of optics, amplitudes of the electromagnetic field at the two different polarizations or wavelengths. In the latter case, the variable $t$ is not time, but rather the propagation distance along the optical waveguide(s). The counterpart of the norm (2) is the expression

$$
P \equiv \frac{1}{2} \int_{-\infty}^{+\infty}\left[\left|\psi_{1}(x)\right|^{2}+\left|\psi_{2}(x)\right|^{2}\right] d x,
$$

which is a dynamical invariant of the continuum model.

The nonlinearity coefficients $g_{j j}(j=1,2)$ and $g_{12}$ account for the intraspecies and inter-species interactions, respectively. In the context of BECs, they are proportional to the respective scattering lengths of interactions between atoms, while in optics they are coefficients of the self-phase modulation (SPM) and cross-phase modulation (XPM), respectively. A positive (negative) scattering length corresponds to the repulsive (attractive) interaction, and results in $g>0$ ( $g$ $<0)$ in Eq. (1) and Eq. (3). Importantly, both the magnitude and sign of the intra-species scattering lengths can be controlled, via the Feshbach resonance, by an external spatially uniform magnetic [31] or optical [32] field. The magnetic field may also provide a tool for the Feshbach-resonance control of the interspecies scattering strength [9]. In the context of optics, the magnitude and sign of the SPM and XPM coefficients are usually fixed (typically, they are negative, corresponding to self-focusing interactions).

We aim to investigate the case of immiscibility, corresponding to

$$
g_{12}>\sqrt{\left|g_{11} g_{22}\right|}
$$

[33], i.e., with the repulsive cross-interaction between components effectively stronger than the self-interaction in each species; the latter may be either repulsive or attractive. The repulsive cross-interaction is the most generic case in BECs, and it occurs in nonlinear optics too (self-defocusing case). The combination of the interspecies repulsion with either repulsion or attraction inside each species is quite possible in the BEC context. In ordinary optical materials, the signs of the XPM and SPM coefficients are identical, i.e., a combination of the self-defocusing XPM and self-focusing SPM is not possible; however, it may be engineered in nonlinear photonic crystals [34].

The trapping potential in Eq. (3) is assumed to be of the usual parabolic type, customarily used in BEC experiments $[1,2]$ :

$$
V(x)=\frac{1}{2} \Omega^{2} x^{2} .
$$

The frequency $\Omega$ measures the strength of the trap.

We will look for stationary solutions to Eq. (1) and Eq. (3) in the forms, respectively,

$$
\begin{gathered}
\psi_{1, n}(t)=e^{i \Lambda_{1} t} u_{n}, \quad \psi_{2, n}(t)=e^{i \Lambda_{2} t} v_{n}, \\
\psi_{1}(x, t)=e^{i \Lambda_{1} t} u(x), \quad \psi_{2}(x, t)=e^{i \Lambda_{2} t} v(x) .
\end{gathered}
$$

Solutions of the boundary-value problems obtained by the substitution of Eqs. (8) in Eq. (3) [or, in the discrete model, by the substitution of Eqs. (7) in Eq. (1)] are found as fixed points of a numerical iteration scheme on a finite-difference grid. Then the linear-stability analysis is performed by assuming a perturbed solution,

$$
\begin{aligned}
& \psi_{1}=e^{i \Lambda_{1} t}\left[u(x)+\epsilon\left(a(x) e^{-\lambda t}+b(x) e^{-\lambda^{*} t}\right)\right], \\
& \psi_{2}=e^{i \Lambda_{2} t}\left[v(x)+\epsilon\left(c(x) e^{-\lambda t}+d(x) e^{-\lambda^{*} t}\right)\right],
\end{aligned}
$$

where $\epsilon$ is the amplitude of infinitesimal perturbations, $\lambda$ is a (generally, complex) eigenvalue (the asterisk stands for complex conjugation), to be found from the linearized version of Eq. (3) along with the corresponding eigenfunctions $\left\{a(x), b(x)^{*}\right\}$ and $\left\{c(x), d(x)^{*}\right\}$. A similar ansatz is used in the 
discrete case, with $x$ substituted by the index $n$.

In the computations, a lattice or interval of sufficiently large size is used in order to ensure that boundary effects do not affect the results. Neumann boundary conditions are typically used.

\section{THE DISCRETE MODEL}

\section{A. Bright-bright soliton pairs}

The first type of localized states that was examined in this context are pairs of bright solitons (one in each component) with a DW between them. This class of solutions is illustrated in Fig. 1 for a typical example corresponding to a weakly immiscible system (which is a realistic case for mixtures of two different spin states $[4,6]$ ) with $g_{11}=g_{22}=-1$ and $g_{12}=1.1$; see Eq. (5). Solution branches were continued starting from the anticontinuum (AC) limit, $C=0$, where the solution was taken as $u_{n}=\delta_{n,-n_{0}}$ and $v_{n}=\delta_{n, n_{0}}$. For this initial configuration we took $\Lambda_{1}=\Lambda_{2}=1$, even though asymmetric solutions can be constructed as well, by taking $\Lambda_{1} \neq \Lambda_{2}$.

First, for $n_{0}=2$ (i.e., with the initial separation $\Delta n \equiv 2 n_{0}$ $-1=3$ between the bright solitons in the two components), we have found that this branch is stable for $C<1.85$. As may be expected, the repulsion between the pulses for larger $C$ (i.e., approaching the continuum limit) overcomes the pinning of the pulses by the substrate lattice potential and causes the configuration to disappear, without allowing one to continue this solution branch to $C \rightarrow \infty$. More specifically, it disappears at $C=1.89$ in a saddle-node bifurcation with another solution, which is initiated in the AC limit as $u_{n}$ $=\delta_{n,-n_{0}}+\delta_{n,-n_{0}-1}$ and $v_{n}=\delta_{n, n_{0}}+\delta_{n, n_{0}+1}$, and is unstable in its existence region for all values of $C$.

The evolution of an unstable solution pertaining to $C$ $=1.86$ is depicted in Fig. 2. It is observed that the solitons settle at a new (roughly constant) distance $\Delta n$ between them, stabilizing themselves this way.

Starting with a larger value of $n_{0}$ in the AC limit (hence, with a larger initial separation $\Delta n$ ) results in a solution that exists and is stable for larger values of $C$; e.g., for $n_{0}=3$, the pair remains stable up to $C=2.34$. We have also studied the effect of the inter-species repulsion strength, by changing $g_{12}$. The result was that the larger $g_{12}$, the instability sets in earlier; e.g., for $g_{12}=2$, the pair becomes unstable at $C$ $=1.75$.

\section{B. Dark-dark soliton pairs}

We now proceed to the case of repulsion in each component (while the interspecies interaction remains repulsive and stronger than the intraspecies repulsion). Actually, it is the most typical case for two different spin states in BECs $[3,4]$.

First, assuming $g_{11}=g_{22}=1$ and $g_{12}=1.1$, we initialize our fixed point numerical scheme in the AC limit, using $u_{n<n_{1}<n_{0}}=-1, u_{n_{1}}=0, u_{n_{1}<n \leqslant n_{0}}=1$, and $u_{n>n_{0}}=0$, the initial ansatz for $v_{n}$ being the mirror image of $u_{n}$ with respect to $n_{0}$. It is easy to see that this configuration corresponds to two dark solitons with centers at $n= \pm n_{1}$, separated by the DW set at $n_{0}=0$, i.e., a solution that may be naturally expected in the present case.
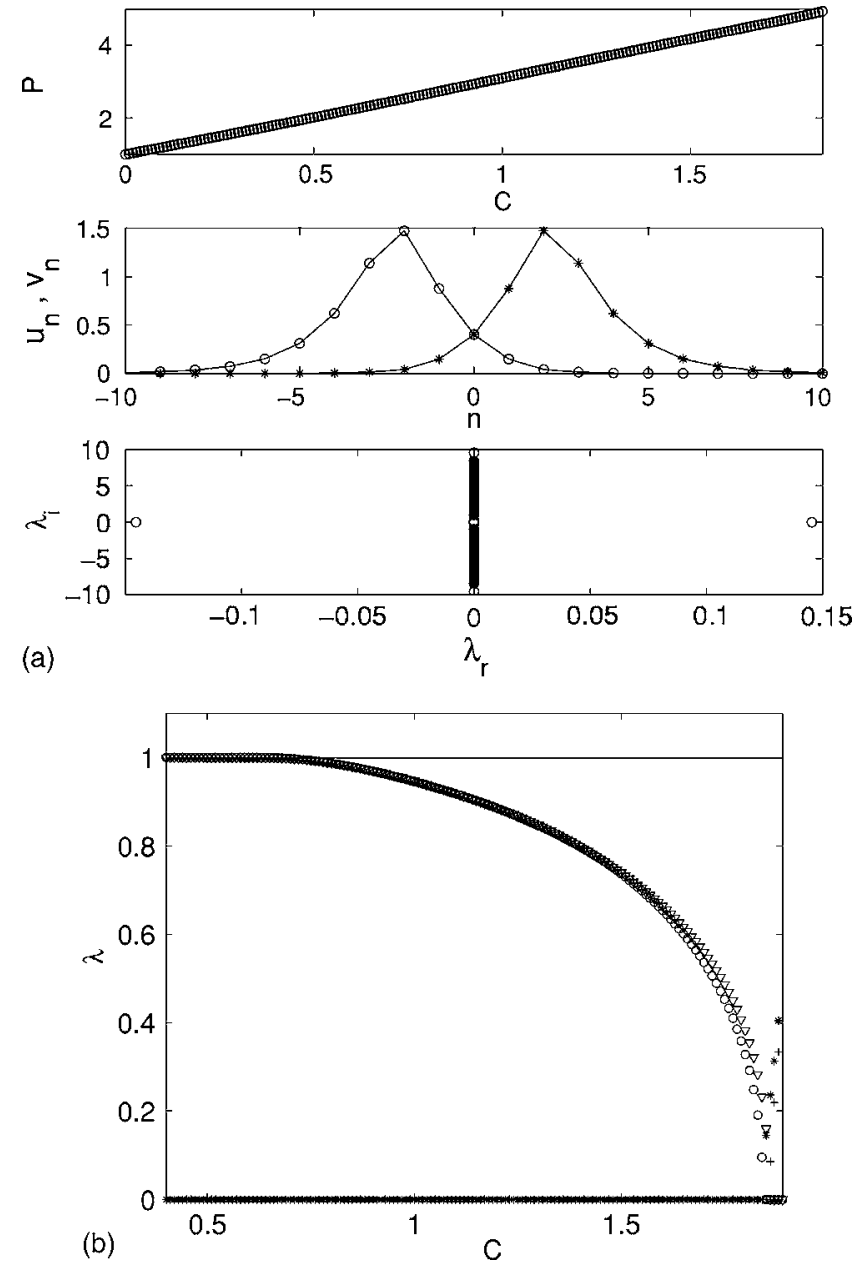

FIG. 1. Discrete bright-bright soliton pair separated by a domain wall. The top panel shows the norm of the solution, $P$ [defined as per Eq. (2)], as a function of $C$; the second panel shows a spatial profile of the two components for $C=1.85$, where the solution becomes unstable due to the presence of a real eigenvalue pair (additional unstable pairs appear at larger $C$ ). The third panel shows the spectral plane $\left(\lambda_{r}, \lambda_{i}\right)$ for the eigenvalues, $\lambda=\lambda_{r}+i \lambda_{i}$. The bottom panel shows the principal eigenvalues bifurcating for $C \approx 0.655$ from the continuous spectrum and approaching the origin of the spectral plane. They become unstable at $C \approx 1.85$ (the second pair becomes real at $C \approx 1.86$ ), destabilizing the solution. The circles and triangles show the eigenvalues when they are still imaginary (stable), while the stars and crosses show them when they are real, i.e., unstable.

In Fig. 3, we show a configuration of this type with $n_{0}$ $=0, n_{1}=-9$. We have found that such solutions may only be stable where a single discrete dark soliton is stable [35], and they are unstable for larger values of $C$ due to two oscillatory instabilities accounted for by two quartets of eigenvalues (see the third panel in Fig. 3). As will be shown below, the dark-dark soliton pair has a larger chance to be stable in the continuum model.

In the last two panels of Fig. 3, where the evolution of an unstable solution is shown (for $C=1$ ), it is observed that the onset of the instability sets the dark solitons into motion. It is worth noting that, although, as mentioned above, the instability threshold of the dark-dark soliton pair is the same as of 

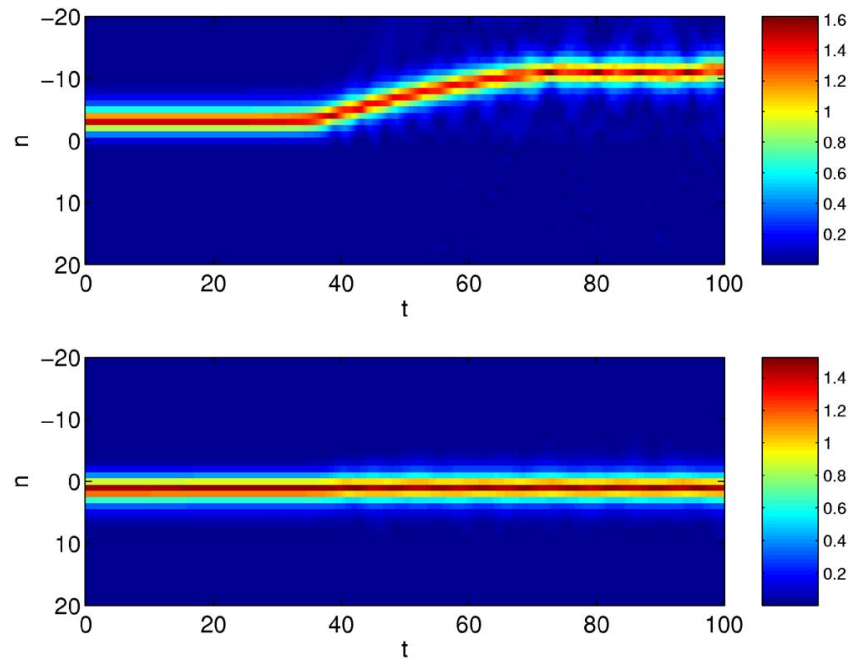

FIG. 2. (Color online) The evolution of an unstable pair of discrete bright solitons separated by the domain wall is shown for $C$ $=1.86$ by means of space-time contour plots of the absolute values of the wave functions, $\left|\psi_{1, n}\right|$ and $\left|\psi_{2, n}\right|$ (top and bottom panels). The solution is perturbed by its most unstable eigenmode.

the single dark soliton, the feature of instability-induced motion occurs only in the two-component model, as an obvious consequence of the repulsion between the two components.

\section{Bright-dark soliton pairs}

We now address hybrid configurations, in which one component supports a bright solitary wave, while the other one supports a dark solitary wave, with a domain wall arising at their interface. Such configurations are possible when $g_{11} g_{22}<0$, as may be the case for BEC mixtures composed by different atom species; an example is the ${ }^{39} \mathrm{~K}-{ }^{87} \mathrm{Rb}$ mixture, in which the potassium (rubidium) features attractive (repulsive) intraspecies interaction, i.e., $g_{22}<0, g_{11}>0$, while the interspecies interaction is repulsive $\left(g_{12}>0\right)$ $[8,9,12]$.

First, assuming that $g_{11}=-g_{22}=1$ and $g_{12}=1.1$, we show an example of a bright-dark soliton pair in Fig. 4. In this case, following the same notation as in the previous subsections, we take $n_{0}=1$ and $n_{1}=-4$ for the dark soliton and $n_{0}$ $=1$ for the bright one.

Generally speaking, the stability domain of the brightdark pair is restricted by the parametric range where the discrete dark soliton is stable by itself. Figure 4 displays the situation near the border of this stability region (for $C$ $=0.07$ ). A difference from the bright-bright and dark-dark soliton pairs considered above is that the hybrid configuration cannot be continued to large values of $C$. This is presumably related to the fact that a part of the pattern develops a "staggered" structure, with a sign-alternating dependence between sites; obviously, staggered structures do not have a continuum limit. We note in passing that, for this reason, we will not attempt to study the continuum counterparts of such structures in the next section.

The bottom two panels of Fig. 4 show the evolution of an unstable bright-dark soliton solution for $C=0.08$, where the
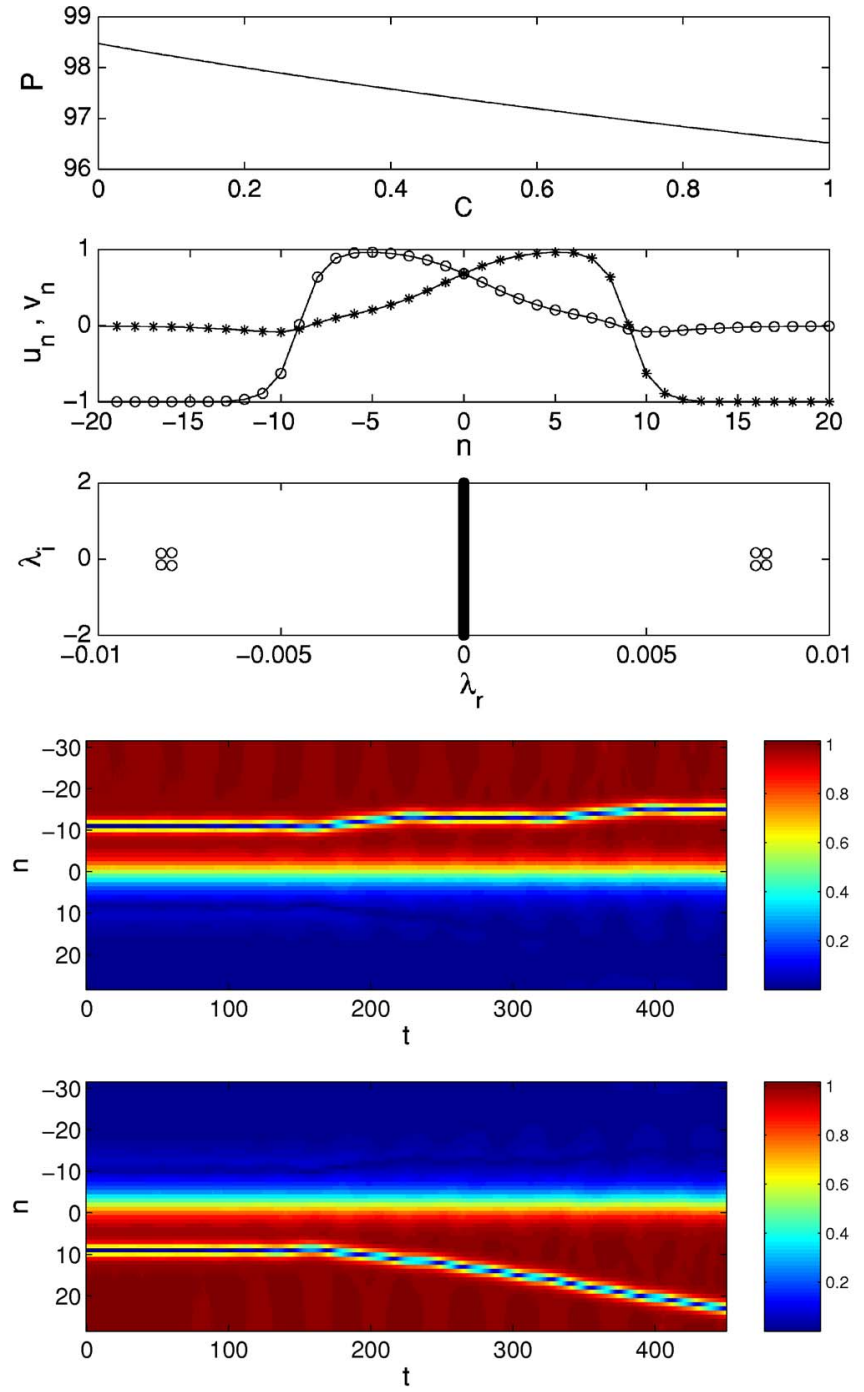

FIG. 3. (Color online) Discrete dark-dark soliton pair separated by a domain wall. The top three panels are similar to the respective ones in Fig. 1; however, the second and third panels correspond to $C=1$. Notice the two unstable quartets in the third panel (their precise location depends on the number of points used in the discretization; for these panels, we have used a 200-site lattice). The last two panels show (through the space-time contour plots of $\left|\psi_{1, n}\right|$ and $\left.\left|\psi_{2, n}\right|\right)$ the evolution of the unstable solution for $C=1$. The solution is perturbed by its most unstable eigenmode.

dark soliton clearly decays. In the simulation, the effect of the instability can be seen after a rather long time $t \approx 1500$ (which is natural, given the very weak coupling between sites which delays the instability manifestation).

\section{THE CONTINUUM MODEL WITH THE EXTERNAL TRAP}

\section{A. Bright-bright soliton pairs}

We now proceed to the model based on the two coupled continuum NLS equations (3). Here, the external parabolic potential due to the presence of a magnetic trap (instead of 

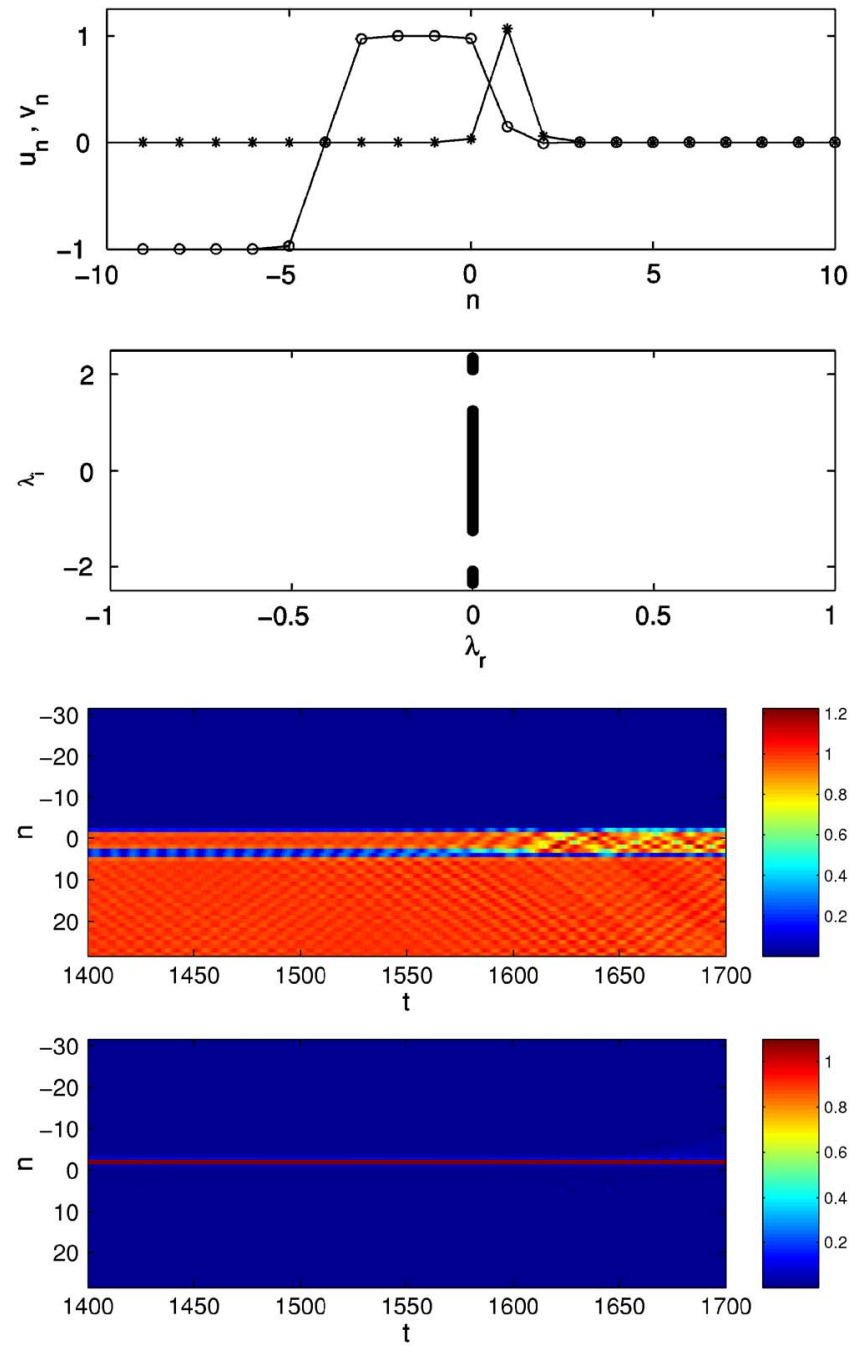

FIG. 4. (Color online) Discrete bright-dark soliton pair separated by a domain wall. The coupling parameter value for this example is $C=0.07$, i.e., slightly below the stability border of the dark solitary wave. The second panel illustrates the stability of the configuration by showing the spectral plane of eigenvalues. The last two panels depict the evolution of an unstable bright-dark soliton for $C=0.08$.

the periodic OL potential which induced the discreteness in the previous section) provides for the force which balances the inter-pulse repulsion and prevents indefinite separation between the components. An example of a stable brightbright soliton pair with the DW nested in the middle is given in Fig. 5. We have found this branch of solutions to be linearly stable (and robust in direct simulations) for all the values of the trap strength $\Omega$ shown in the figure.

In Fig. 5, we also show the separation $\Delta$ between the two bright solitons as a function of the trap strength $\Omega$. Naturally, this dependence is a monotonically decreasing one: $\Delta$ should go to infinity as $\Omega \rightarrow 0$, when the force balancing the intercomponent repulsion vanishes. On the other hand, as $\Omega$ becomes very large, each one of the pulses gets strongly confined at the trap's center.

As shown in detail in Appendix A, we have used a variational approximation (VA) to tackle this problem analyti-
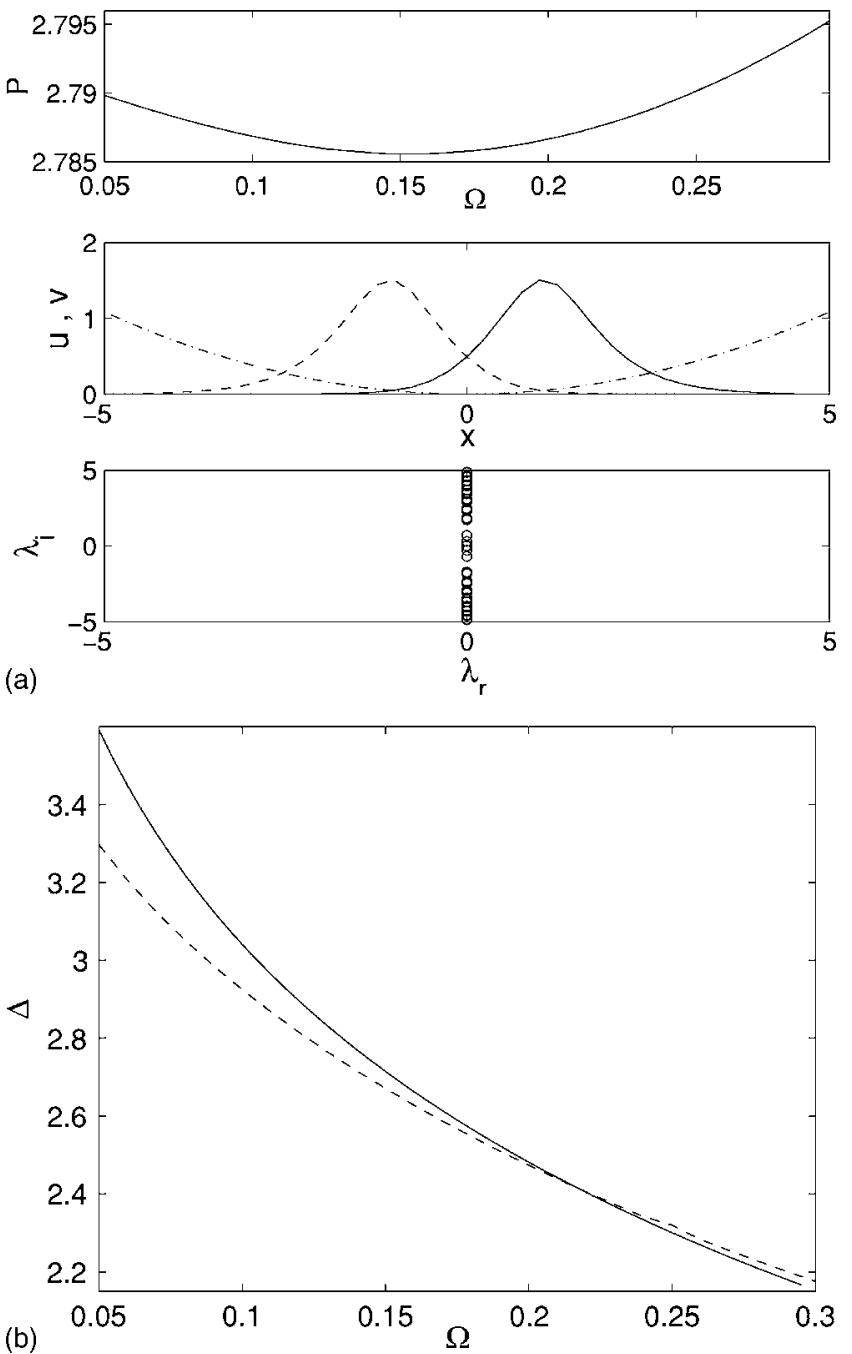

(b)

FIG. 5. Continuous bright-bright soliton pair separated by a domain wall. The top panel shows the solution's norm $P$ [defined as per Eq. (4)] as a function of $\Omega$. The second panel shows the spatial profile of the solution (for the two components in solid and dashed line, respectively; the external potential is shown by dash-dotted line) for $\Omega=0.3$. The third panel shows the stability of this solution through the corresponding eigenvalues for $\Omega=0.3$ (no real pair). The bottom panel of the figure illustrates the result of continuation for the separation $\Delta$ between the pulses of this stable branch of solutions, as a function of the magnetic trap strength $\Omega$. The numerical result is given by the solid line while the variational prediction (see text and Appendix A) is shown by the dashed line.

cally. The VA was based on a Gaussian ansatz,

$$
\{u(x), v(x)\}=\pi^{-1 / 4} \sqrt{\frac{P}{W}} \exp \left(-\frac{1}{2}\left(\frac{x \mp \frac{\Delta}{2}}{W}\right)^{2}\right),
$$

for the spatial profile of the solution [see Eqs. (8)], which allows the width $W$, separation $\Delta$ between the pulses, and solution's (half-)norm $P$ [the latter is defined as per Eq. (4)] to be free variational parameters. The VA leads to a system of three algebraic equations for the relevant parameters. We have tested this analytical prediction (the dashed line in the 


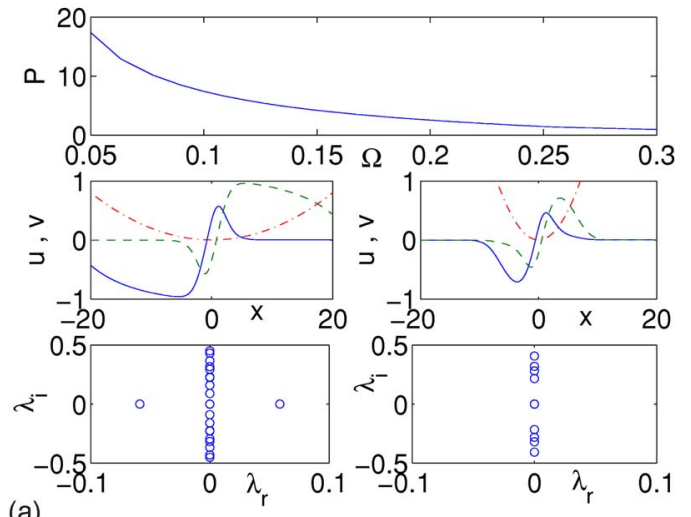

(a)
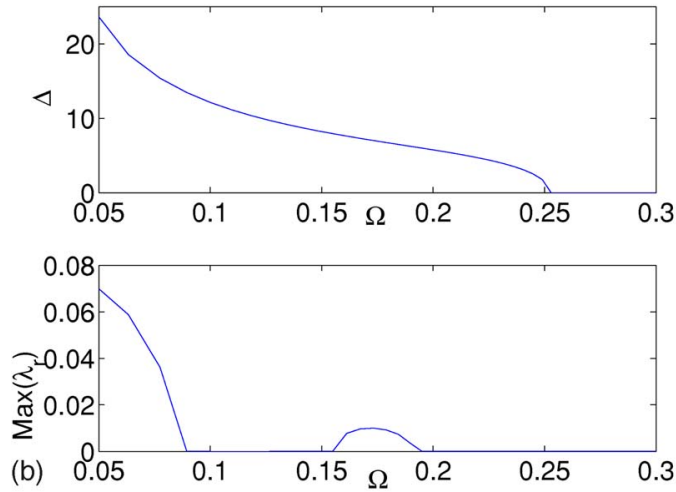

FIG. 6. (Color online) Continuous dark-dark soliton pair with an embedded domain wall. The top panel shows the continuation of the solution's norm vs the trap's strength $\Omega$. For the next panels, the four subplots show the profiles (top) and stability (bottom) of the solution for $\Omega^{2}=0.004$ (left) and $\Omega^{2}=0.04$ (right). The last two panels show, respectively, the separation between centers of mass of the two components, $\Delta$, and the real part of the most unstable eigenvalue of the configuration, $\lambda_{r}$, vs $\Omega$. It is clearly observed that the two dark solitons merge for $\Omega>0.25$. The solution is stable in two distinct intervals: $0.09<\Omega<0.155$ and $\Omega>0.195$.

bottom panel of Fig. 5) against the corresponding numerical result (the solid line), obtaining a fairly good agreement. For small $\Omega$, the solitons are well separated, hence the difference between the strong localization of the Gaussian ansatz (10) and weaker localization of the exact soliton based on the sech waveform becomes essential: obviously, the force of repulsion between Gaussians decays with $\Delta$ faster than between sech pulses. This may explain a larger discrepancy between the VA-predicted and numerically found values of $\Delta$ at smaller values of $\Omega$.

\section{B. Dark-dark soliton pairs}

Bound pairs of dark solitons with an embedded DW have also been investigated in the continuum model with the interspecies and intraspecies repulsion and external trap. A typical example is shown in Fig. 6 for $g_{11}=g_{22}=1$ and $g_{12}$ $=2$. The figure presents the continuation of this solution branch as a function of the trap's strength $\Omega$. Intervals of the values of $\Omega$ where such solutions are linearly stable have been identified: $0.09<\Omega<0.155$ and $\Omega>0.195$. It is noteworthy that there exist two disjoint stability regions. As the

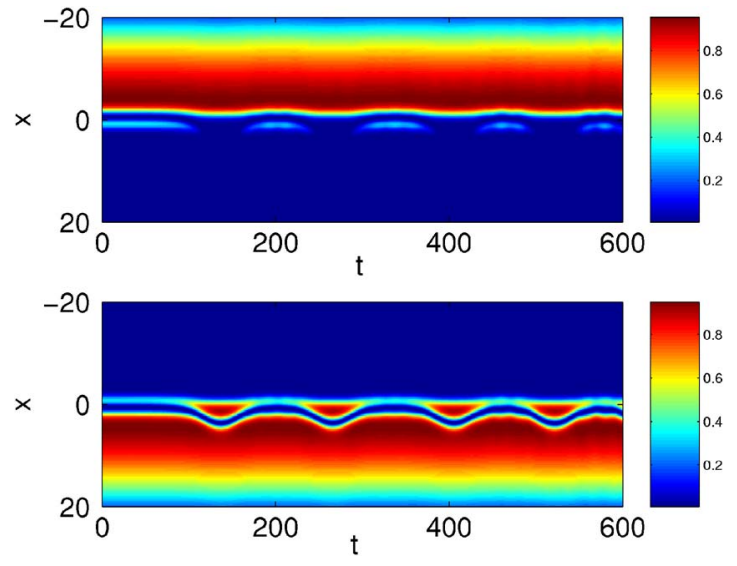

FIG. 7. (Color online) The two panels show the space-time contour plot of the square modulus of the two fields for $g_{12}=2=2 g_{11}$ $=2 g_{22}$ and for $\Omega^{2}=0.004$.

trap strength further increases, it gradually presses the two dark soliton pair closer to each other, eventually leading to a merger of the two components into a single antisymmetric profile at $\Omega>0.25$.

We have investigated the evolution of unstable dark soliton pairs in direct simulations. A typical example is shown in Fig. 7 for $\Omega^{2}=0.004$. Periodic oscillations of the two-soliton structure arise (as a result of the instability) between the initial state and an asymmetric one, in which the dark soliton is far more pronounced in one of the components.

\section{BRIGHT SOLITON PAIRS IN THE SYSTEM WITH LINEAR COUPLING}

Another setting where we have found bright soliton pairs with the embedded DW due to the cross repulsion is the system in which linear coupling between the components plays the role of the attractive force that balances their mutual repulsion (cf. Ref. [26]):

$$
\begin{aligned}
& i \partial_{t} \psi_{1}=\left(-\frac{1}{2} \partial_{x}^{2}-\left|\psi_{1}\right|^{2}+g_{12}\left|\psi_{2}\right|^{2}\right) \psi_{1}-\kappa \psi_{2}, \\
& i \partial_{t} \psi_{2}=\left(-\frac{1}{2} \partial_{x}^{2}+g_{12}\left|\psi_{1}\right|^{2}-\left|\psi_{2}\right|^{2}\right) \psi_{2}-\kappa \psi_{1},
\end{aligned}
$$

where we set $g_{11}=g_{22}=-1, g_{12}$ is assumed to be positive and $\kappa$ is the rate of the linear interconversion (coupling) between the species. The trapping potential is not included, in anticipation of the possibility that the linear coupling can support solitons without the external trap.

Similarly to the above model with the parabolic trap, the VA based on ansatz (10) can be applied here, leading to algebraic equations relating the width, power, and separation between centers of the waves in the different components. The equations are given in Appendix B. A noteworthy prediction of the VA is that solutions with a nonzero pulse separation, $\Delta>0$, exist for $g_{12}>1 / 3$ (see the Appendix B for details), a feature that we check below against numerical results.

Figure 8 illustrates the profiles and stability of these solutions for $\kappa=1$. The main issue that arises in this case is that 

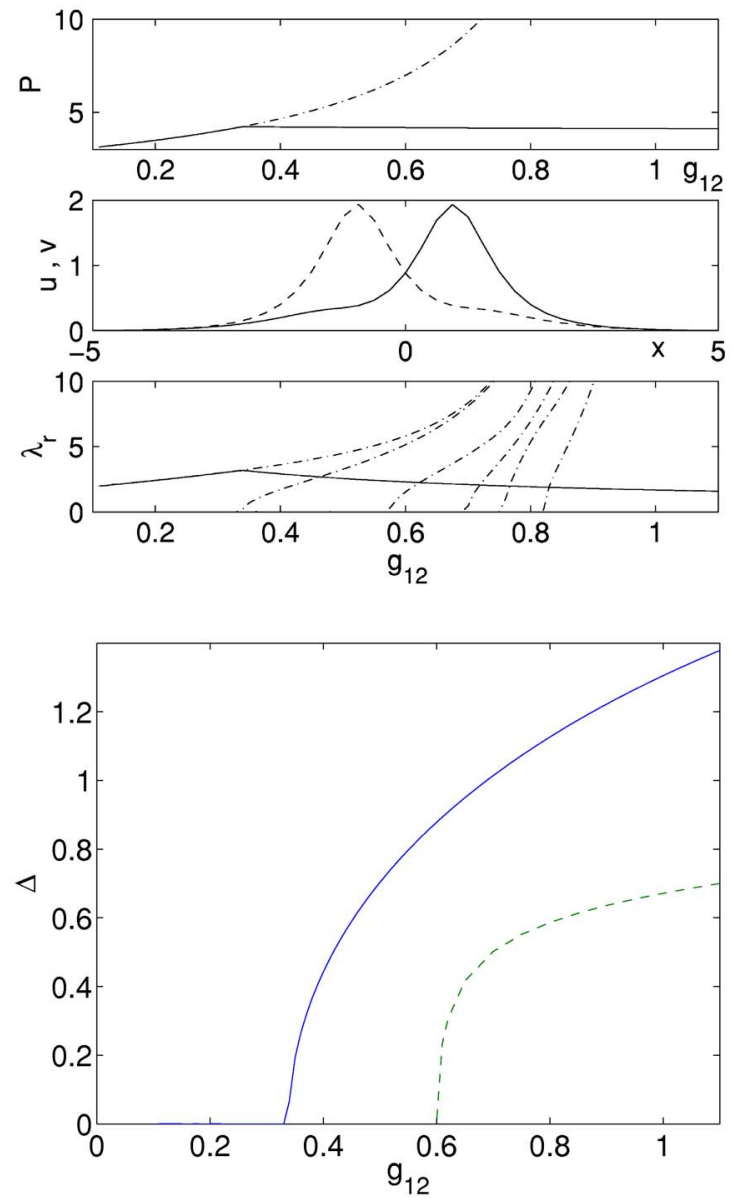

FIG. 8. (Color online) Continuous bright-bright soliton pair bound by linear coupling. The top panel shows the norm of the solution as a function of the coefficient $g_{12}$ which accounts for the nonlinear repulsion (the parameter controlling the linear coupling is fixed to $\kappa=1)$. The dash-dotted line shows the branch with zero separation between the solitons $(\Delta=0)$. The two branches merge for $g_{12} \leqslant 1 / 3$. The second panel shows an example of the bright-soliton pair with the domain wall between them, for $g_{12}=1.1$. The third panel shows the dependence of the most unstable (real) eigenvalues for the $\Delta \neq 0$ (solid line) and the $\Delta=0$ (dash-dotted line) solutions. The bottom panel shows the pulse separation between the solitons in the bound-pair solution as a function of $g_{12}$. The solid and dashed lines, respectively, depict the numerical results and prediction of the variational approximation (see Appendix B).

the branch of solutions with $\Delta \neq 0$ is always unstable due to a pair of real eigenvalues. We have compared the separation between the solitons in the pair as obtained numerically and with the VA prediction (see bottom panel in Fig. 8). While the VA captures the trend of the relevant dependence, it is less successful in its quantative comparison due to the distorted nature of the pulse profile (cf. second panel of Fig. 8). Notice also that the branch of solutions with the nonvanishing separation ceases to exist, merging with the branch of $\Delta=0$ solutions, at $g_{12} \leqslant 1 / 3$. The latter branch (with $\Delta=0$ ) is unstable for all values of $g_{12}$ shown in the figure. In fact, it has multiple unstable eigenvalues (see third panel in Fig. 8) which gradually disappear; the merger of the two branches occurs when only one real eigenvalue pair is left. Let us also

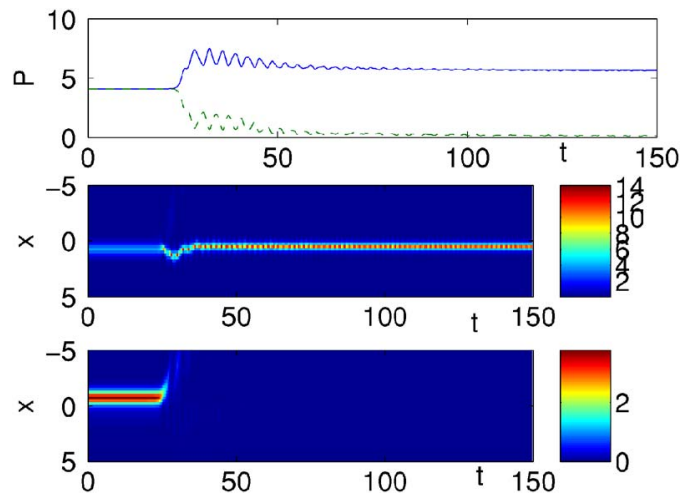

FIG. 9. (Color online) Evolution of a bright-soliton pair in the model with the linear coupling, for $g_{12}=1.1$ and $\kappa=1$. The solution is perturbed only by numerical noise, which is sufficient to provoke its very rapid transformation into a breathing pulse in one component, while the other one keeps practically no matter. The top panel shows the norm of each component as a function of time. The middle and lower panels show the evolution in terms of space-time contour plots of the square modulus of the two fields.

note, in passing, that we have also tried to fix $g_{12}$ and vary $\kappa$, however, we were not successful in stabilizing this branch of solutions in the latter setting either.

The evolution of the unstable bright soliton pairs in the presence of the linear coupling was monitored in direct simulations, as shown in Fig. 9. It is seen that the solution rapidly breaks its symmetry, and collects nearly all the matter in one of the two components, while the other one becomes practically empty. A more careful study of these asymmetric solutions (superimposed bright solitons with different norms) reveals that they are always stable, when they emerge, bifurcating through a pitchfork bifurcation that destabilizes the symmetric branch of solutions (where both components carry bright solitons centered around $x=0$ ). Notice that the $g_{12}=0$ analog of this bifurcation picture was analyzed extensively in the previous work of [36] and subsequently in the variational study of [37]. The main difference in the presence of interspecies coupling concerns the exact location of the bifurcation point; this question, however, is beside the scope of the present study, focusing on the phenomenology of vector solitary waves with embedded domain walls; as such, it will be deferred for a future publication. A branch of the asymmetric solutions (centered around $x=0$ ) for fixed $\kappa=1$ is depicted in Fig. 10.

Other solutions have also been obtained in the model with the linear coupling, such as the weakly unstable asymmetric bright soliton pairs shown in Fig. 11. Their instability involves three eigenvalue pairs, and their evolution is displayed in the bottom two panels of the figure. We observe that the instability sets in for $t>100$, leading to a slightly asymmetric partition of matter between the components. Eventually, at longer times, after a collision-type event and some exchange of matter, the solitons separate indefinitely.

\section{CONCLUSIONS}

In this work, we have studied several types of vector (two-component) solitons in a system of coupled discrete 

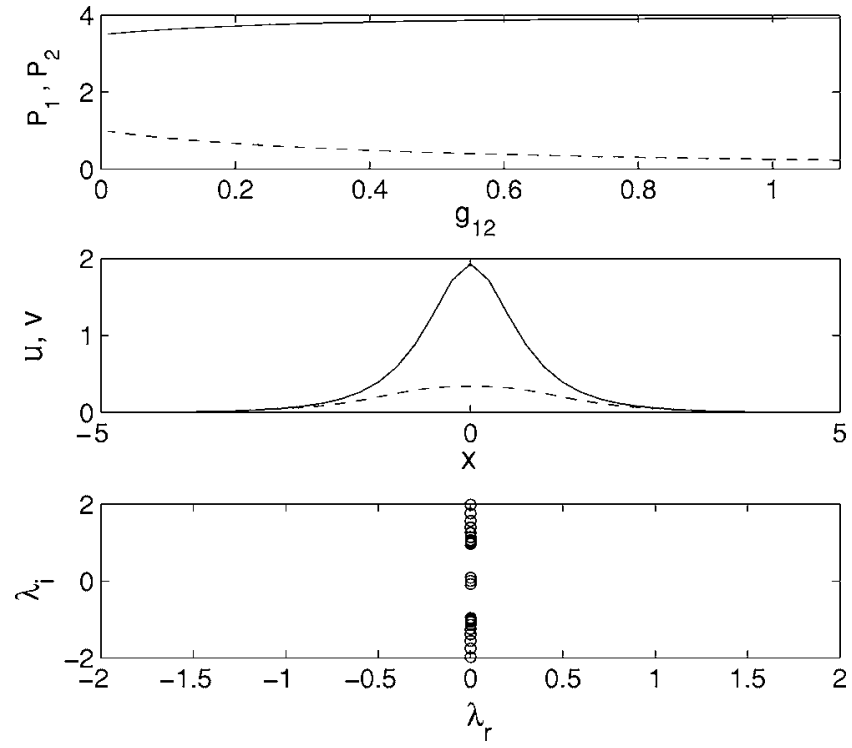

FIG. 10. Branch of the stable asymmetric bright soliton pair in the presence of linear coupling. The top panel depicts the norm of the bright solitons in each component as $g_{12}$ is varied. This branch is found to be stable in all of its domain. The middle and bottom panels show an example of such a configuration and its stability spectrum for $g_{12}=1$. The linear coupling for these results is fixed at $\kappa=1$.

and continuous nonlinear Schrödinger (NLS) equations. A distinctive feature of the localized states is the presence of a domain wall (DW) embedded between the two components, due to the nonlinear repulsion between them. The models are relevant to binary mixtures of Bose-Einstein condensates (BECs) with the repulsive interspecies interaction, and may also find applications in nonlinear optics (especially, in photonic crystals). In the BEC mixture with attractive (repulsive) intraspecies interactions, the relevant configuration consists of a bright (dark) soliton in each component, which are separated by the DW. Hybrid configurations, with a bright soliton in the one component and a dark one in the other, were also found for a mixture of self-attractive and self-repulsive BECs.

We have found that such configurations can be stable in a variety of settings. Bright soliton pairs have a linear-stability region in the discrete model, which appertains to the BEC mixtures trapped in very deep optical lattices. In the continuum limit, they are robust too in the presence of a confining potential, which is a typical ingredient of BEC experiments. Dark-soliton pairs behave quite differently, featuring alternating regimes of stability and instability. Evolution of generic unstable configurations was monitored by dint of direct simulations. The continuum model with the linear coupling was investigated too; in that case, bright soliton pairs exist without any trapping potential, as the linear-attraction force can balance the nonlinear repulsion.

The states predicted in this work can be created in realistic BEC experiments. To this effect, sufficiently strong interspecies repulsion (facilitating the formation of the DW) may be provided, tuning the inter-species interactions by means
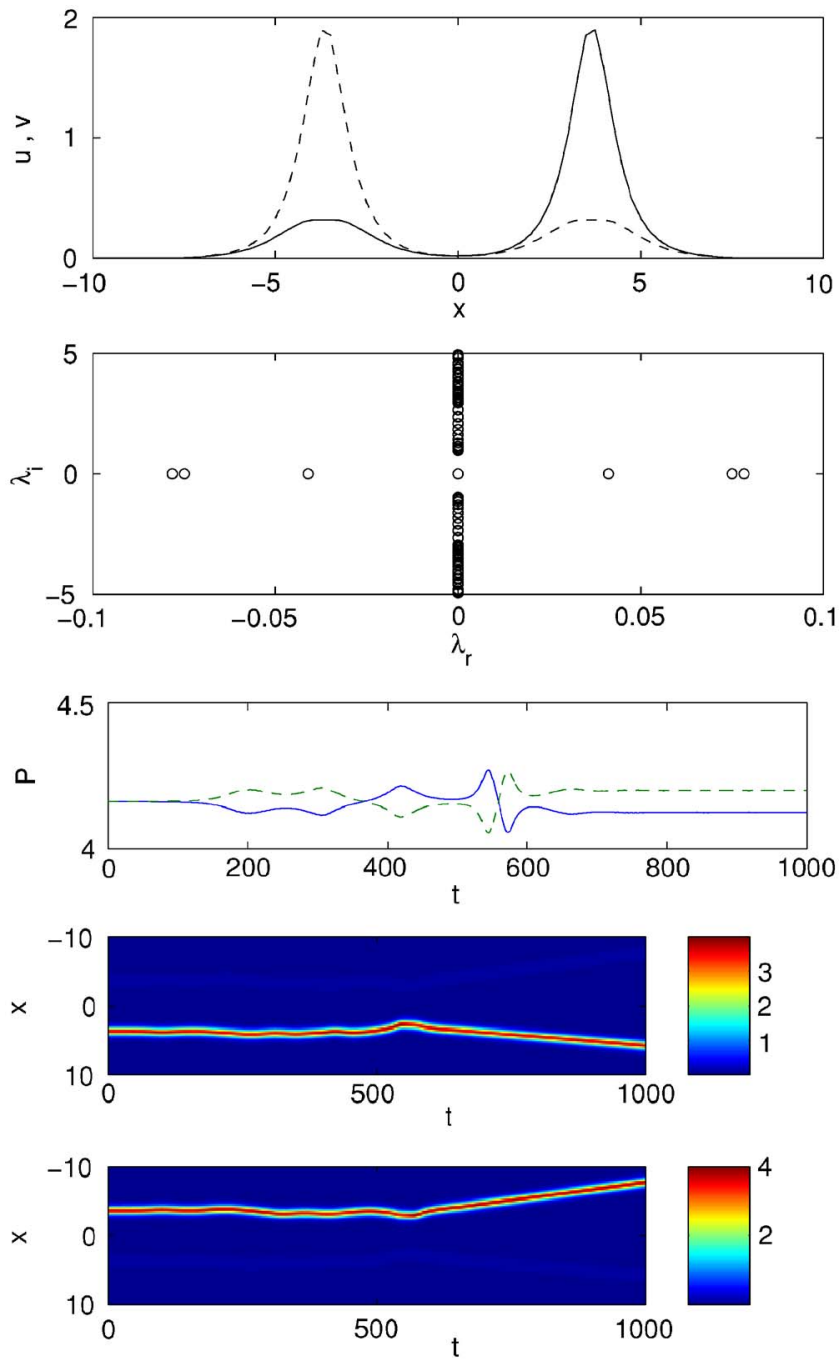

FIG. 11. (Color online) Weakly unstable asymmetric brightsoliton pair for $g_{12}=1.1$ and $\kappa=1$. The top panel is the solution profile, and the second panel illustrates its (in)stability. The third panel shows the evolution of the norms of the two components as a function of time. The two bottom panels show space-time contour plots of square modulus of the two fields.

of the Feshbach resonance [9,31]. In the presently available experimental settings, such as mixtures of two different spin states in ${ }^{87} \mathrm{Rb}[3,4]$ or ${ }^{23} \mathrm{Na}$ [5], the configurations which are easiest to implement, among ones considered in this work, are the pairs of dark solitons (or, possibly, dark-bright ones for the $\mathrm{K}-\mathrm{Rb}$ mixtures [12]), which clearly seem within reach in optical lattices [2] or in the magnetic trap [1,2]. On the other hand, while bright solitons have been created in one-component BECs [38], the generation of bright-soliton pairs appears to be a more challenging possibility in the currently available systems. However, a similar and more straightforward possibility may be provided by pairs of bright solitons of the gap type, which were recently created in a repulsive BEC loaded in an optical lattice [39]. The latter possibility is currently under study and will be reported elsewhere. 


\section{ACKNOWLEDGMENTS}

P.G.K. gratefully acknowledges support from NSF-DMS0204585, NSF-CAREER. R.C.G. and P.G.K. also acknowledge support from NSF-DMS-0505663. B.A.M. appreciates support from the Israel Science Foundation through Grant No. 8006/03. H.S. acknowledges support from the Royal Netherlands Academy of Arts and Sciences (KNAW).

\section{APPENDIX A: THE VARIATIONAL APPROXIMATION FOR THE CONTINUUM MODEL} (3),

We start with the symmetric version of the system of Eqs.

$$
\begin{aligned}
& i \partial_{t} \psi_{1}=\left(-\frac{1}{2} \partial_{x}^{2}-\left|\psi_{1}\right|^{2}+g\left|\psi_{2}\right|^{2}+\frac{\Omega^{2}}{2} x^{2}\right) \psi_{1}, \\
& i \partial_{t} \psi_{2}=\left(-\frac{1}{2} \partial_{x}^{2}+g\left|\psi_{1}\right|^{2}-\left|\psi_{2}\right|^{2}+\frac{\Omega^{2}}{2} x^{2}\right) \psi_{2},
\end{aligned}
$$

in which we have set $g_{11}=g_{22}=1$ and $g_{12} \equiv g>0$ (to provide for repulsion between the two species). The control parameters of the model are $g$ and the norms of the solution,

$$
P=\int_{-\infty}^{+\infty}\left|\psi_{1}(x)\right|^{2} d x=\int_{-\infty}^{+\infty}\left|\psi_{2}(x)\right|^{2} d x
$$

(for the symmetric case under consideration). Symmetric stationary solutions are to be looked for as

$$
\psi_{1}=\exp (-i \mu t) u(x), \quad \psi_{2}=\exp (-i \mu t) v(x),
$$

with a real chemical potential $\mu$. The real functions $v(x)$ and $u(x)$ satisfy the equations

$$
\begin{aligned}
& \mu u=-\frac{1}{2} u^{\prime \prime}+\left(-u^{2}+g v^{2}\right) u+\frac{\Omega^{2}}{2} x^{2} u, \\
& \mu u=-\frac{1}{2} v^{\prime \prime}+\left(-v^{2}+g u^{2}\right) v+\frac{\Omega^{2}}{2} x^{2} v .
\end{aligned}
$$

Stationary equations (A4) can be derived from the Lagrangian $L=\int_{-\infty}^{+\infty} \mathcal{L} d x$, with the density

$$
\begin{aligned}
\mathcal{L}= & \frac{1}{4}\left[\left(\frac{d u}{d x}\right)^{2}+\left(\frac{d v}{d x}\right)^{2}\right]-\frac{\mu}{2}\left(u^{2}+v^{2}\right)-\frac{1}{4}\left(u^{4}+v^{4}\right)+\frac{g}{2} u^{2} v^{2} \\
& +\frac{1}{4} \Omega^{2} x^{2}\left(u^{2}+v^{2}\right) .
\end{aligned}
$$

To apply the variational approximation (VA), we use the ansatz (10), defined in the text. Its substitution in Eq. (A5) and integration yield

$$
\begin{aligned}
L= & \frac{1}{4} \frac{P}{W^{2}}-\mu P-\frac{1}{2 \sqrt{2 \pi}} \frac{P^{2}}{W}+\frac{g}{2 \sqrt{2 \pi}} \frac{P^{2}}{W} \exp (-\epsilon) \\
& +\frac{1}{4} \Omega^{2} P W^{2}(\epsilon+1),
\end{aligned}
$$

where a separation parameter has been defined,

$$
\epsilon \equiv \Delta^{2} / 2 W^{2} .
$$

One may now consider $P$ as an unknown that must be found for given $\mu$. Then, for given $\mu$ and $g$, the variational parameters are $P, W$, and $\epsilon$. The variational equation $\partial L / \partial \epsilon$ $=0$ takes the form

$$
\exp (-\epsilon)=\Omega^{2} \frac{\sqrt{2 \pi} W^{3}}{2 g P},
$$

and the equation $\partial L / \partial W=0$ amounts to

$$
\frac{1}{\sqrt{2 \pi}} P W+\Omega^{2}\left(\frac{1}{2}+\epsilon\right) W^{4}=1 .
$$

Finally, the equation $\partial L / \partial P=0$ yields an expression for the chemical potential

$$
\mu=\frac{1}{4 W^{2}}-\frac{1}{\sqrt{2 \pi}} \frac{P}{W}+\frac{1}{4} \Omega^{2} W^{2}(3+\epsilon),
$$

which also can be used to predict the condition for the absence of instability of the solution accounted for by real eigenvalues in the form of the Vakhitov-Kolokolov (VK) criterion, $d \mu / d P<0$ [40].

\section{APPENDIX B: VARIATIONAL APPROXIMATION FOR THE CONTINUUM MODEL WITH THE LINEAR COUPLING}

To apply the VA to the system of linearly coupled equations (11) we set, by means of a rescaling, $\kappa=1$. Then, in addition to $g_{12}$, a control parameter of the model is the norm of the solution, $P$, defined as per Eq. (4). Searching for stationary solutions of Eqs. (11) in the form of Eqs. (A3), we then arrive at equations

$$
\begin{aligned}
& \mu V=-\frac{1}{2} V^{\prime \prime}+\left(-V^{2}+g_{12} U^{2}\right) V-V \\
& \mu U=-\frac{1}{2} U^{\prime \prime}+\left(-U^{2}+g_{12} V^{2}\right) U-V .
\end{aligned}
$$

We will look for stationary solutions by varying $P$, for a fixed nonlinearity coefficient $g_{12}$. We may assume that, for small $P$, centers of both components coincide, so that $u(x)$ $=v(x)$. With the increase of $P$, the nonlinear repulsion between the species increases, and one should encounter a critical norm, $P_{\mathrm{cr}}$, at which a transition to a split state, with a finite separation, $\Delta$, between the centers of the components (it is precisely a transition to a two-component bright soliton with an embedded domain wall). The coordinates of the centers of mass of the two species can be defined as

$$
\{\xi, \eta\}=P^{-1} \int_{-\infty}^{+\infty}\left\{u^{2}(x), v^{2}(x)\right\} x d x .
$$

In the split state, we assume equal norms of the components, with their centers located at $\{\xi, \eta\}= \pm \Delta / 2$.

Proceeding to the VA proper, we notice that stationary equations (B1) can be derived from the Lagrangian $L=\int_{-\infty}^{+\infty} \mathcal{L} d x$, with the density 


$$
\begin{aligned}
\mathcal{L}= & \frac{1}{4}\left[\left(\frac{d u}{d x}\right)^{2}+\left(\frac{d v}{d x}\right)^{2}\right]-\frac{\mu}{2}\left(u^{2}+v^{2}\right)-\frac{1}{4}\left(v^{4}+u^{4}\right) \\
& +\frac{1}{2} g_{12} u^{2} v^{2}-u v .
\end{aligned}
$$

Using again the ansatz given in Eq. (10), we derive the effective Lagrangian in the following form:

$$
\begin{aligned}
L= & -\mu P+\frac{1}{4} \frac{P}{W^{2}}-\frac{1}{2 \sqrt{2 \pi}} \frac{P^{2}}{W}+\frac{g_{12}}{2 \sqrt{2 \pi}} \frac{P^{2}}{W} \exp (-\epsilon) \\
& -P \exp (-2 \epsilon),
\end{aligned}
$$

where $\epsilon$ is the same as in Eq. (A7). Assuming, as in Appendix A, $P$ as an unknown that must be found for given $\mu$ and $g_{12}$, the variational equations yield the following results:

$$
\begin{gathered}
\exp (-2 \epsilon)=\frac{2 \sqrt{2 \pi} W}{g_{12} P}, \\
-\frac{1}{W}+\frac{P}{2 \sqrt{2 \pi}}-\frac{2 \sqrt{2 \pi} W^{2}}{g_{12} P}=0, \\
\mu=\frac{1}{4 W^{2}}-\frac{P}{2 \sqrt{2 \pi} W}-\frac{\sqrt{2 \pi} W}{g_{12} P} .
\end{gathered}
$$

The latter equation may be used to predict the stability of the states as per the above-mentioned VK criterion, i.e., $d \mu / d P<0$.

For given $P$, it is necessary to find $W$ from Eq. (B6), and then find $\Delta$ from Eqs. (B5) and (A7). Physical solutions must have $W$ real and positive, and $\exp (-2 \epsilon) \leqslant 1$. For $P \rightarrow 0$, Eq. (B6) take the form $W^{3} \approx-g_{12} P /(\sqrt{2 \pi})$, i.e., all the solutions are unphysical (one negative, and two complex conjugate, which actually implies that the VA does not apply for $P$ $\rightarrow 0$ ). Equation (B6), obviously, does not admit a real root crossing zero, therefore one root (which is negative and real for $P \rightarrow 0$ ) always remains real and negative, i.e., unphysical. With the increase of $P$, a pair of physical roots for $W$ appear when the complex roots merge and thus bifurcate into a real pair. Analysis shows that this happens at

$$
P=P_{\min }=3 \sqrt{\pi}\left(3 g_{12}\right)^{-1 / 4},
$$

the corresponding physical root itself being

$$
W\left(P=P_{\min }\right)=\left(\frac{3 g_{12}}{4}\right)^{1 / 4}
$$

(it is a double root at $P=P_{\min }$ ). The substitution of (B8) and (B9) in Eq. (B5) yields an expression that determines the value of the separation parameter $\epsilon$ corresponding to $P$ $=P_{\text {min }}$ :

$$
\exp (4 \epsilon)=3 g_{12}
$$

Finally, Eq. (B10) yields a physical solution, with $\exp (-2 \epsilon)$ $\leqslant 1$, if $g_{12} \geqslant 1 / 3$.
[1] F. Dalfovo, S. Giorgini, L. P. Pitaevskii, and S. Stringari, Rev. Mod. Phys. 71, 463 (1999); C. J. Pethick and H. Smith, BoseEinstein Condensation in Dilute Alkali Gases (Cambridge University Press, Cambridge, UK, 2002); L. P. Pitaevskii and S. Stringari, Bose-Einstein Condensation (Clarendon Press, Oxford, 2003).

[2] F. Dalfovo, S. Giorgini, L. P. Pitaevskii, and S. Stringari, Rev. Mod. Phys. 71, 463 (1999); P. G. Kevrekidis and D. J. Frantzeskakis, Mod. Phys. Lett. B 18, 173 (2004); V. A. Brazhnyi and V. V. Konotop, Mod. Phys. Lett. B 18, 627 (2004); P. G. Kevrekidis, R. Carretero-González, D. J. Frantzeskakis, and I. G. Kevrekidis, Mod. Phys. Lett. B 18, 1481 (2004).

[3] C. J. Myatt, E. A. Burt, R. W. Ghrist, E. A. Cornell, and C. E. Wieman, Phys. Rev. Lett. 78, 586 (1997).

[4] D. S. Hall, M. R. Matthews, J. R. Ensher, C. E. Wieman, and E. A. Cornell, Phys. Rev. Lett. 81, 1539 (1998).

[5] D. M. Stamper-Kurn, M. R. Andrews, A. P. Chikkatur, S. Inouye, H.-J. Miesner, J. Stenger, and W. Ketterle, Phys. Rev. Lett. 80, 2027 (1998).

[6] H. Pu and N. P. Bigelow, Phys. Rev. Lett. 80, 1130 (1998).

[7] H. Pu and N. P. Bigelow, Phys. Rev. Lett. 80, 1134 (1998).

[8] G. Ferrari, M. Inguscio, W. Jastrzebski, G. Modugno, G. Roati, and A. Simoni, Phys. Rev. Lett. 89, 053202 (2002).

[9] A. Simoni, F. Ferlaino, G. Roati, G. Modugno, and M. Ingus- cio, Phys. Rev. Lett. 90, 163202 (2003).

[10] M. J. Jamieson, H. Sarbazi-Azad, H. Ouerdane, G.-H. Jeung, Y. S. Lee, and W. C. Lee, J. Phys. B 36, 1085 (2003).

[11] D. Schumayer and B. Apagyi, Phys. Rev. A 69, 043620 (2004).

[12] G. Modugno, G. Ferrari, G. Roati, R. J. Brecha, A. Simoni, and M. Inguscio, Science 294, 1320 (2001); G. Modugno, M. Modugno, F. Riboli, G. Roati, and M. Inguscio, Phys. Rev. Lett. 89, 190404 (2002).

[13] M. Mudrich, S. Kraft, K. Singer, R. Grimm, A. Mosk, and M. Weidemüller, Phys. Rev. Lett. 88, 253001 (2002).

[14] T.-L. Ho and V. B. Shenoy, Phys. Rev. Lett. 77, 3276 (1996).

[15] B. D. Esry, C. H. Greene, J. P. Burke, Jr., and J. L. Bohn, Phys. Rev. Lett. 78, 3594 (1997).

[16] Th. Busch, J. I. Cirac, V. M. Pérez-García, and P. Zoller, Phys. Rev. A 56, 2978 (1997); R. Graham and D. Walls, Phys. Rev. A 57, 484 (1998); B. D. Esry and C. H. Greene, Phys. Rev. A 57, 1265 (1998).

[17] M. Trippenbach, K. Goral, K. Rzazewski, B. Malomed, and Y. B. Band, J. Phys. B 33, 4017 (2000).

[18] S. Coen and M. Haelterman, Phys. Rev. Lett. 87, 140401 (2001).

[19] B. A. Malomed, H. E. Nistazakis, D. J. Frantzeskakis, and P. G. Kevrekidis, Phys. Rev. A 70, 043616 (2004); Math. Com- 
put. Simul. 69, 400 (2005).

[20] Th. Busch and J. R. Anglin, Phys. Rev. Lett. 87, 010401 (2001).

[21] P. Öhberg and L. Santos, Phys. Rev. Lett. 86, 2918 (2001).

[22] P. G. Kevrekidis, H. E. Nistazakis, D. J. Frantzeskakis, B. A. Malomed, and R. Carretero-Gonzalez, Eur. Phys. J. D 28, 181 (2004).

[23] B. Deconinck, P. G. Kevrekidis, H. E. Nistazakis, and D. J. Frantzeskakis, Phys. Rev. A 70, 063605 (2004).

[24] M. A. Porter, P. G. Kevrekidis, and B. A. Malomed, Physica D 196, 106 (2004).

[25] J. Williams, R. Walser, J. Cooper, E. Cornell, and M. Holland, Phys. Rev. A 59, R31 (1999); P. Öhberg and S. Stenholm, Phys. Rev. A 59, 3890 (1999); D. T. Son and M. A. Stephanov, Phys. Rev. A 65, 063621 (2002); S. D. Jenkins and T. A. Brian Kennedy, Phys. Rev. A 68, 053607 (2003); Q. Han Park and J. H. Eberly, Phys. Rev. A 70, 021602(R) (2004).

[26] M. I. Merhasin, B. A. Malomed, and R. Driben, J. Phys. B 38, 877 (2005)

[27] A. Trombettoni and A. Smerzi, Phys. Rev. Lett. 86, 2353 (2001).

[28] F. Kh. Abdullaev, B. B. Baizakov, S. A. Darmanyan, V. V. Konotop, and M. Salerno, Phys. Rev. A 64, 043606 (2001).

[29] G. L. Alfimov, P. G. Kevrekidis, V. V. Konotop, and M. Salerno, Phys. Rev. E 66, 046608 (2002).

[30] J. Hudock, P. G. Kevrekidis, B. A. Malomed, and D. N. Christodoulides, Phys. Rev. E 67, 056618 (2003).

[31] S. Inouye, M. R. Andrews, J. Stenger, H.-J. Miesner, D. M. Stamper-Kurn, and W. Ketterle, Nature (London) 392, 151 (1998); J. Stenger, S. Inouye, M. R. Andrews, H.-J. Miesner, D. M. Stamper-Kurn, and W. Ketterle, Phys. Rev. Lett. 82, 2422 (1999); J. L. Roberts, N. R. Claussen, J. P. Burke, Jr., C.
H. Greene, E. A. Cornell, and C. E. Wieman, Phys. Rev. Lett. 81, 5109 (1998); S. L. Cornish, N. R. Claussen, J. L. Roberts, E. A. Cornell, and C. E. Wieman, Phys. Rev. Lett. 85, 1795 (2000); E. A. Donley, N. R. Claussen, S. L. Cornish, J. L. Roberts, E. A. Cornell, and C. E. Wieman, Nature (London) 412, 295 (2001).

[32] P. O. Fedichev, Yu. Kagan, G. V. Shlyapnikov, and J. T. M. Walraven, Phys. Rev. Lett. 77, 2913 (1996); M. Theis, G. Thalhammer, K. Winkler, M. Hellwig, G. Ruff, R. Grimm, and J. H. Denschlag, Phys. Rev. Lett. 93, 123001 (2004).

[33] V. P. Mineev, Zh. Eksp. Teor. Fiz. 67, 263 (1974) [Sov. Phys. JETP 40, 132 (1974)].

[34] N. A. R. Bhat and J. E. Sipe, Phys. Rev. E 64, 056604 (2001); S. I. Inoue and Y. Aoyagi, Phys. Rev. Lett. 94, 103904 (2005).

[35] M. Johansson and Yu. S. Kivshar, Phys. Rev. Lett. 82, 85 (1999).

[36] N. Akhmediev and J. M. Soto-Crespo, Phys. Rev. E 49, 4519 (1994).

[37] B. A. Malomed, I. M. Skinner, P. L. Chu, and G. D. Peng, Phys. Rev. E 53, 4084 (1996).

[38] K. E. Strecker, G. B. Partridge, A. G. Truscott, and R. G. Hulet, Nature (London) 417, 150 (2002); L. Khaykovich, F. Schreck, G. Ferrari, T. Bourdel, J. Cubizolles, L. D. Carr, Y. Castin, and C. Salomon, Science 296, 1290 (2002).

[39] B. Eiermann, Th. Anker, M. Albiez, M. Taglieber, P. Treutlein, K.-P. Marzlin, and M. K. Oberthaler, Phys. Rev. Lett. 92, 230401 (2004).

[40] M. G. Vakhitov and A. A. Kolokolov, Izv. Vyssh. Uchebn. Zaved., Radiofiz. 16, 1020 (1973) [Radiophys. Quantum Electron. 16, 783 (1973)]; L. Bergé, Phys. Rep. 303, 260 (1998). 